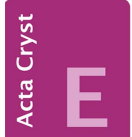
COMMUNICATIONS

ISSN 2056-9890

\section{Crystal structure of 1,3,6,8-tetrabromo- 9-ethyl-9H-carbazole}

\author{
Mykola Bezuglyi, ${ }^{\mathrm{a}, \mathrm{b} *}$ Gintare Grybauskaite, ${ }^{\mathrm{b}}$ Gintautas \\ Bagdziunas $^{\mathrm{b}}$ and Juozas Vidas Grazulevicius ${ }^{\mathrm{b}}$

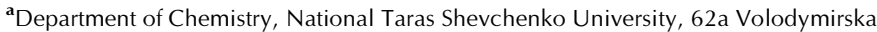 \\ st., Kyiv, Ukraine, and ${ }^{\mathbf{b}}$ Department of Polymer Chemistry and Technology, Kaunas \\ University of Technology, Radvilenu Road 19, LT-50254, Kaunas, Lithuania. \\ *Correspondence e-mail: nikolay_bezugliy@ukr.net
}

Received 16 May 2015; accepted 25 May 2015

Edited by W. T. A. Harrison, University of Aberdeen, Scotland

In the title compound, $\mathrm{C}_{14} \mathrm{H}_{9} \mathrm{Br}_{4} \mathrm{~N}$, the tricyclic ring system is almost planar (r.m.s. deviation for the 13 non- $\mathrm{H}$ atoms $=$ $0.017 \AA$ ) and the methyl $\mathrm{C}$ atom deviates from the mean plane of the ring system by $1.072(17) \AA$. In the crystal, $\mathrm{Br} \cdots \mathrm{Br}$ contacts [3.636 (3) and 3.660 (3) $\AA$ ] slightly shorter than the van der Waals contact distance of $3.70 \AA$ are seen.

Keywords: crystal structure; carbazole; halogen-halogen contact.

CCDC reference: 1402621

\section{Related literature}

For applications of $N$-substituted carbazole derivatives in anticancer research, see: Caulfield et al. (2002). For their use in optoelectronic devices, see: Niu et al. (2011); Miyazaki et al. (2014); Grigalevicius et al. (2002).

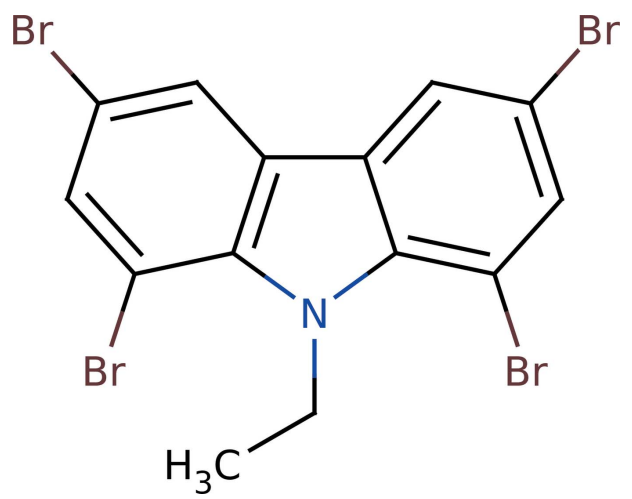

\section{Experimental}

2.1. Crystal data

$\mathrm{C}_{14} \mathrm{H}_{9} \mathrm{Br}_{4} \mathrm{~N}$

$M_{r}=510.85$

Monoclinic, $P 2_{1}$

$a=4.202(2) \AA$

$b=14.654(6) \AA$

$c=12.245(6) \AA$

$\beta=92.758(18)^{\circ}$

$V=753.1(6) \AA^{3}$

$Z=2$

Mo $K \alpha$ radiation

$\mu=10.70 \mathrm{~mm}^{-1}$

$T=293 \mathrm{~K}$

$0.40 \times 0.13 \times 0.12 \mathrm{~mm}$

\subsection{Data collection}

Rigaku XtaLAB mini diffractometer

Absorption correction: multi-scan (REQAB; Rigaku, 1998)

$T_{\min }=0.115, T_{\max }=0.277$

2.3. Refinement

$R\left[F^{2}>2 \sigma\left(F^{2}\right)\right]=0.056$

$w R\left(F^{2}\right)=0.130$

$S=1.01$

2600 reflections

172 parameters

1 restraint

$\mathrm{H}$-atom parameters constrained

2755 measured reflections 2599 independent reflections 2071 reflections with $F^{2}>2.0 \sigma\left(F^{2}\right)$ $R_{\text {int }}=0.021$

Data collection: CrystalClear-SM Expert (Rigaku, 2011); cell refinement: CrystalClear-SM Expert; data reduction: CrystalClear-SM Expert; program(s) used to solve structure: SIR92 (Altomare et al., 1994); program(s) used to refine structure: SHELXL97 (Sheldrick, 2008); molecular graphics: CrystalStructure (Rigaku, 2010); software used to prepare material for publication: CrystalStructure.

\section{Acknowledgements}

This research was supported by FP7 REGPOT-2012-2013-1 ICT project CEOSeR under grant agreement No. 316010.

Supporting information for this paper is available from the IUCr electronic archives (Reference: HB7428).

\section{References}

Altomare, A., Cascarano, G., Giacovazzo, C., Guagliardi, A., Burla, M. C., Polidori, G. \& Camalli, M. (1994). J. Appl. Cryst. $27,435$.

Caulfield, T., Cherrier, M. P., Combeau, C. \& Mailliet, P. (2002). Eur. Patent No. 1253141

Flack, H. D. (1983). Acta Cryst. A39, 876-881.

Grigalevicius, S., Ostrauskaite, J., Grazulevicius, J. V., Gaidelis, V., Jankauskas, V. \& Sidaravicius, J. (2002). Mat. Chem. Phys. 77, 281-284.

Miyazaki, T., Shibahara, M., Fujishige, J., Watanabe, M., Goto, K. \& Shinmyozu, T. (2014). J. Org. Chem. 79, 11440-11453.

Niu, F., Niu, H., Liu, Y., Lian, J. \& Zeng, P. (2011). RSC Adv. 1, 415-423.

Rigaku (1998). REQAB. Rigaku Corporation, Tokyo, Japan.

Rigaku (2010). CrystalStructure. Rigaku Corporation, Tokyo, Japan.

Rigaku (2011). CrystalClear-SM Expert . Rigaku Corporation, Tokyo, Japan. Sheldrick, G. M. (2008). Acta Cryst. A64, 112-122. 


\section{supporting information}

Acta Cryst. (2015). E71, o373 [doi:10.1107/S2056989015010117]

\section{Crystal structure of 1,3,6,8-tetrabromo-9-ethyl-9H-carbazole}

\section{Mykola Bezuglyi, Gintare Grybauskaite, Gintautas Bagdziunas and Juozas Vidas Grazulevicius}

\section{S1. Synthesis and crystallization}

9-Ethyl-9H-carbazole $(0.904 \mathrm{~g}, 4.63 \mathrm{mmol})$ was added to the solution of $N$-bromosuccinimide (NBS) (3.708 g, 20.83 $\mathrm{mmol}$ ) in $30 \mathrm{ml}$ of DMF. The reaction mixture was heated at $60^{\circ} \mathrm{C}$ for 24 hours. When the reaction completed (monitored via TLC) the solution was poured into a large amount of water with ice. The precipitate was filtered off and crystallized from the mixture of isopropanol and DMF (volume ratio about 5:1) to isolate the product as needles. The bulk sample appears yellowish, but individual crystals are colourless. Yield $1.80 \mathrm{~g}(76 \%)$, m.p. $155-156^{\circ} \mathrm{C} .{ }^{1} \mathrm{H}$ NMR (700 MHz, $\left.\mathrm{CDCl}_{3}\right) \delta 7.92(\mathrm{~d}, J=1.8 \mathrm{~Hz}, 2 \mathrm{H}), 7.69(\mathrm{~d}, J=1.8 \mathrm{~Hz}, 2 \mathrm{H}), 5.10(\mathrm{q}, J=7.1 \mathrm{~Hz}, 2 \mathrm{H}), 1.33(\mathrm{t}, J=7.1 \mathrm{~Hz}, 3 \mathrm{H})$.

\section{S2. Refinement}

All hydrogen atoms were placed in geometrically idealized positions and constrained to ride on their parent atoms, with C $-\mathrm{H}=0.930 \AA$ for aromatic $\mathrm{C}-\mathrm{H}$, with $0.969 \AA$ for methylene $\mathrm{C}-\mathrm{H}, 0.957 \AA$ for methyl distances and $\mathrm{U}_{\text {iso }}(\mathrm{H})=1.2$ $\mathrm{U}_{\mathrm{eq}}$. 


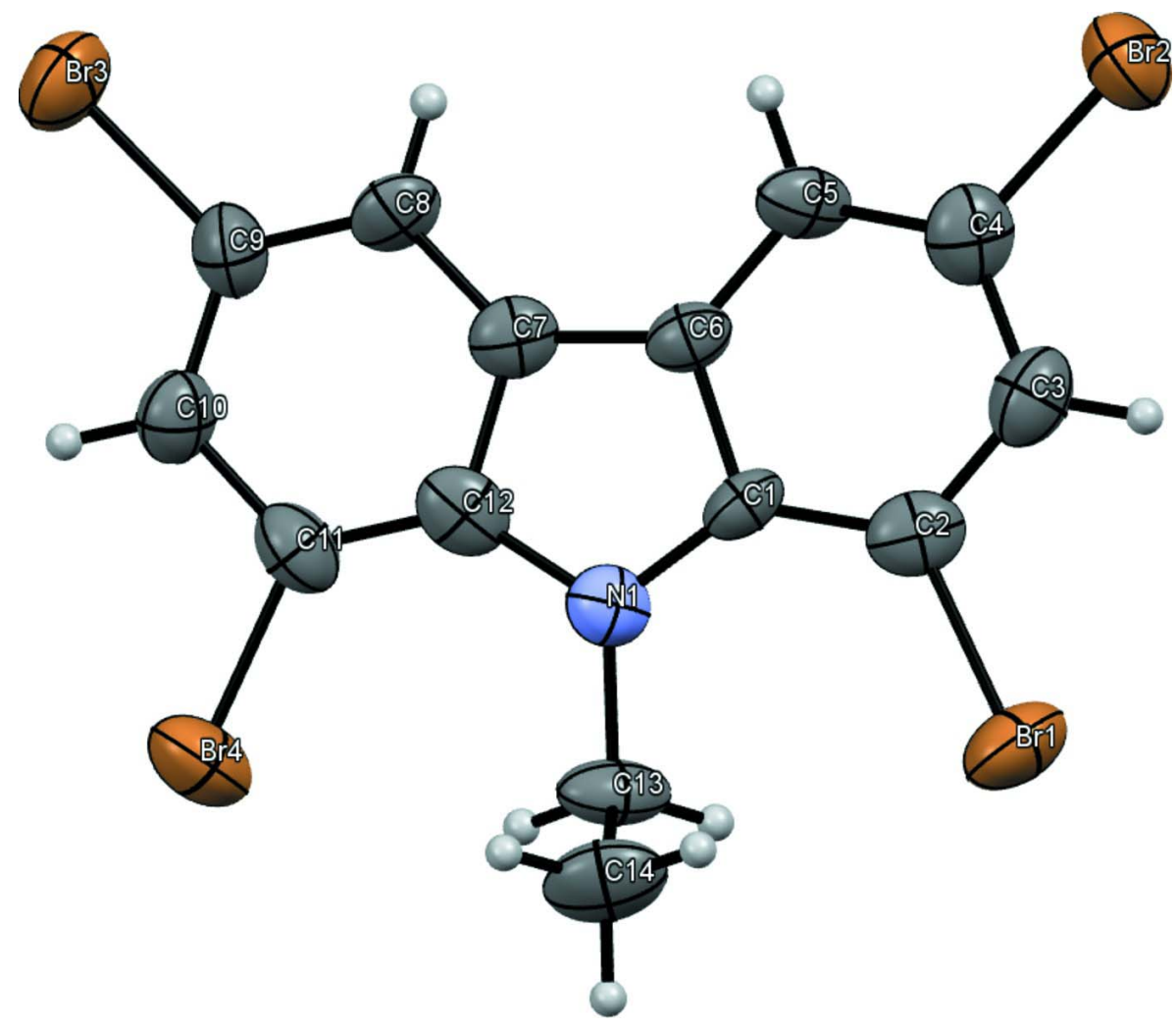

\section{Figure 1}

The molecular structure of the title molecule with displacement ellipsoids drawn at the $50 \%$ probability level.

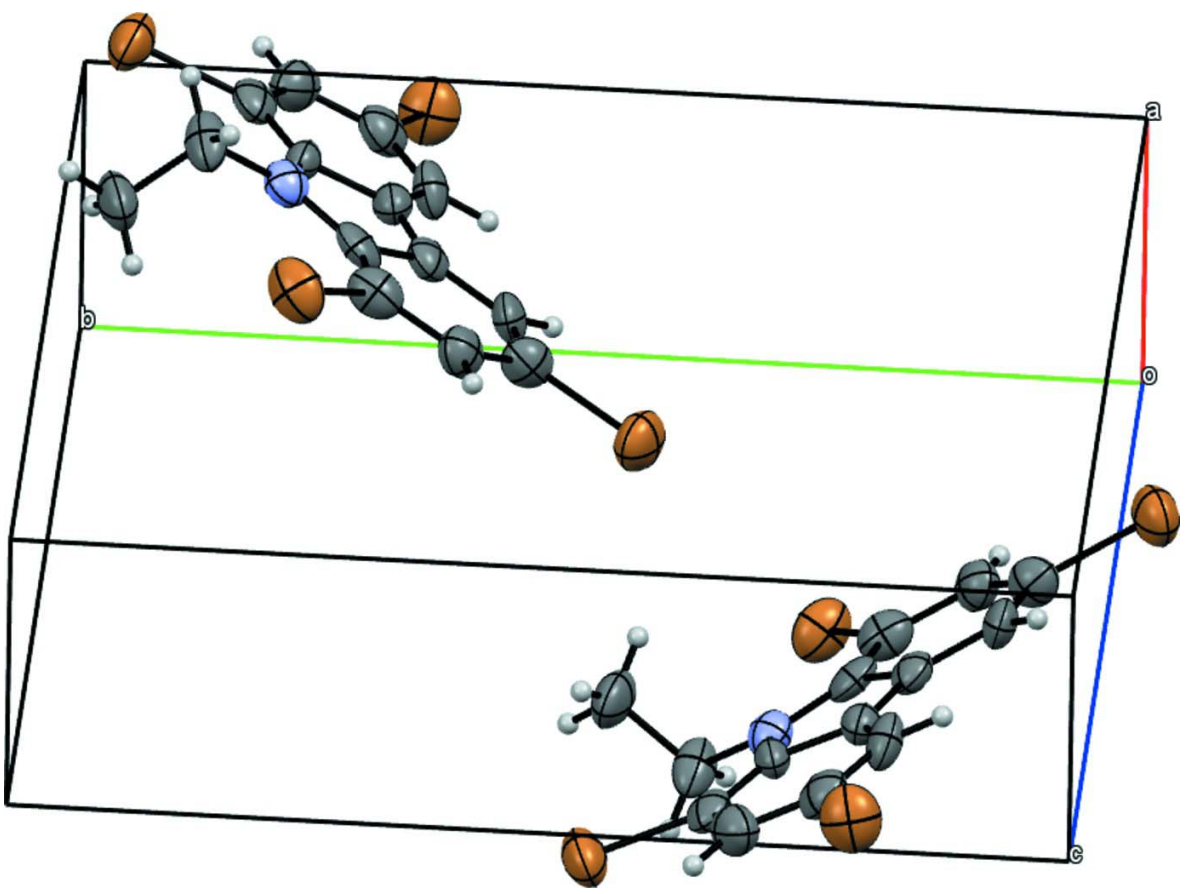

Figure 2

The crystal packing of the title compound. 


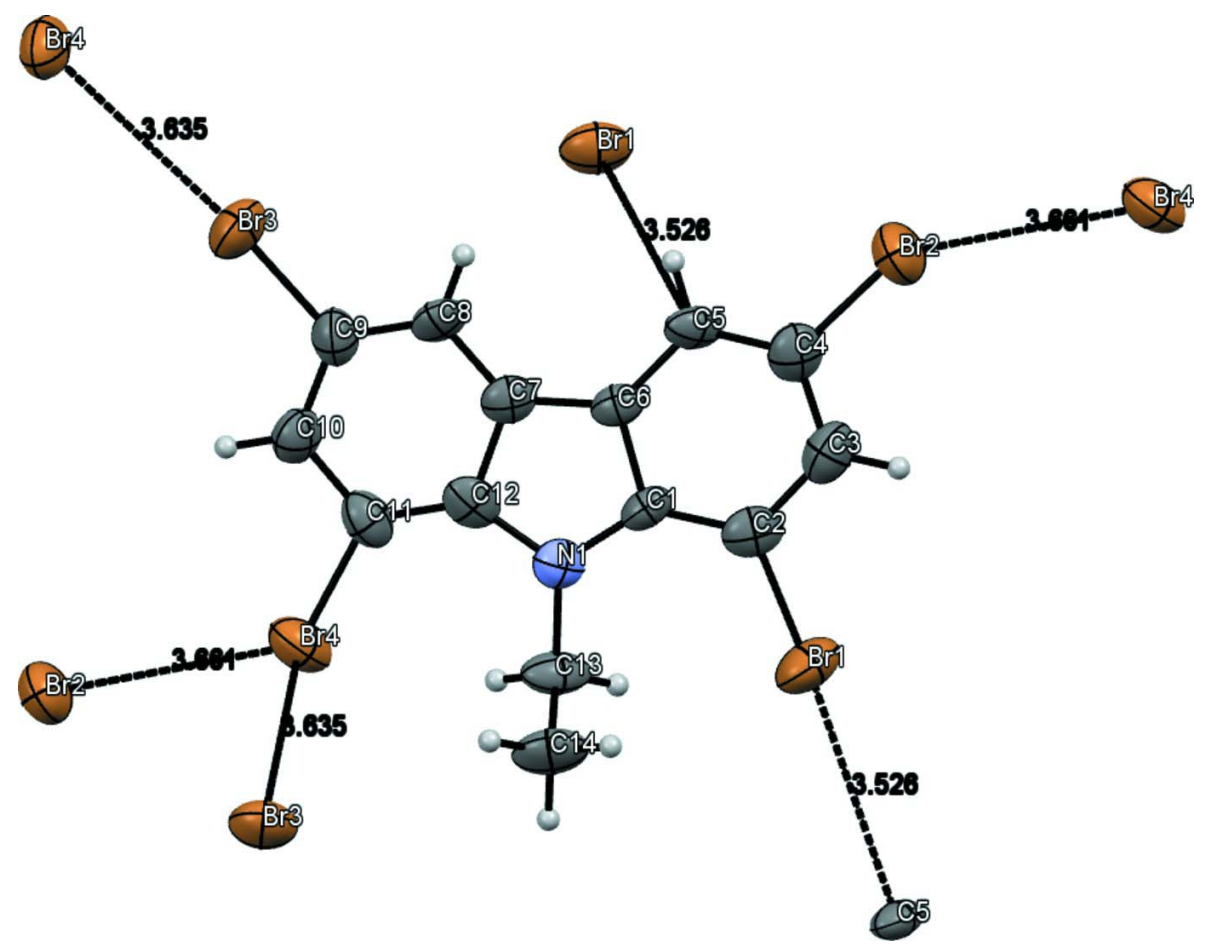

Figure 3

$\mathrm{C}-\mathrm{Br} \cdots \mathrm{Br}$ and $\mathrm{Br} \cdots \pi$ intermolecular contacts.

\section{1,3,6,8-Tetrabromo-9-ethyl-9H-carbazole}

Crystal data

$\mathrm{C}_{14} \mathrm{H}_{9} \mathrm{Br}_{4} \mathrm{~N}$

$M_{r}=510.85$

Monoclinic, $P 2_{1}$

Hall symbol: $\mathrm{P} 2 \mathrm{yb}$

$a=4.202(2) \AA$

$b=14.654(6) \AA$

$c=12.245(6) \AA$

$\beta=92.758(18)^{\circ}$

$V=753.1(6) \AA^{3}$

$Z=2$

\section{Data collection}

Rigaku XtaLAB mini diffractometer

Detector resolution: 6.827 pixels $\mathrm{mm}^{-1}$ $\omega$ scans

Absorption correction: multi-scan

(REQAB; Rigaku, 1998)

$T_{\min }=0.115, T_{\max }=0.277$

2755 measured reflections
$F(000)=480.00$

$D_{\mathrm{x}}=2.253 \mathrm{Mg} \mathrm{m}^{-3}$

Mo $K \alpha$ radiation, $\lambda=0.71075 \AA$

Cell parameters from 2306 reflections

$\theta=3.2-27.5^{\circ}$

$\mu=10.70 \mathrm{~mm}^{-1}$

$T=293 \mathrm{~K}$

Chip, colorless

$0.40 \times 0.13 \times 0.12 \mathrm{~mm}$

2599 independent reflections

2071 reflections with $F^{2}>2.0 \sigma\left(F^{2}\right)$

$R_{\text {int }}=0.021$

$\theta_{\max }=27.5^{\circ}$

$h=-5 \rightarrow 5$

$k=-18 \rightarrow 18$

$l=-15 \rightarrow 4$ 


\section{Refinement}

Refinement on $F^{2}$

$R\left[F^{2}>2 \sigma\left(F^{2}\right)\right]=0.056$

$w R\left(F^{2}\right)=0.130$

$S=1.01$

2600 reflections

172 parameters

1 restraint

Primary atom site location: structure-invariant direct methods

Secondary atom site location: difference Fourier map
Hydrogen site location: inferred from neighbouring sites

$\mathrm{H}$-atom parameters constrained

$w=1 /\left[\sigma^{2}\left(F_{\mathrm{o}}{ }^{2}\right)+(0.0517 P)^{2}\right]$

where $P=\left(F_{\mathrm{o}}^{2}+2 F_{\mathrm{c}}{ }^{2}\right) / 3$

$(\Delta / \sigma)_{\max }<0.001$

$\Delta \rho_{\max }=0.74 \mathrm{e} \AA^{-3}$

$\Delta \rho_{\min }=-0.66$ e $\AA^{-3}$

Absolute structure: Flack (1983), 868 Friedel

Pairs

Absolute structure parameter: 0.05 (4)

Special details

Refinement. Refinement was performed using all reflections. The weighted $R$-factor $(w R)$ and goodness of fit $(S)$ are based on $F^{2}$. $R$-factor (gt) are based on $F$. The threshold expression of $F^{2}>2.0 \sigma\left(F^{2}\right)$ is used only for calculating $R$-factor (gt).

Fractional atomic coordinates and isotropic or equivalent isotropic displacement parameters $\left(\AA^{2}\right)$

\begin{tabular}{lllll}
\hline & $x$ & $y$ & $z$ & $U_{\text {iso }}{ }^{\prime} U_{\text {eq }}$ \\
\hline Br1 & $1.0896(3)$ & $0.54414(9)$ & $0.00371(12)$ & $0.0652(4)$ \\
$\mathrm{Br} 2$ & $0.4335(4)$ & $0.80930(10)$ & $-0.24666(11)$ & $0.0710(4)$ \\
$\mathrm{Br} 3$ & $0.3881(3)$ & $1.05176(9)$ & $0.38945(12)$ & $0.0657(4)$ \\
$\mathrm{Br} 4$ & $1.0717(3)$ & $0.73324(10)$ & $0.49105(11)$ & $0.0654(4)$ \\
$\mathrm{N} 1$ & $0.9560(19)$ & $0.7062(6)$ & $0.2062(8)$ & $0.041(2)$ \\
$\mathrm{C} 1$ & $0.854(3)$ & $0.7148(7)$ & $0.0995(9)$ & $0.037(3)$ \\
$\mathrm{C} 2$ & $0.893(3)$ & $0.6617(8)$ & $0.0045(10)$ & $0.046(3)$ \\
$\mathrm{C} 3$ & $0.768(3)$ & $0.6924(8)$ & $-0.0998(10)$ & $0.051(3)$ \\
$\mathrm{C} 4$ & $0.598(3)$ & $0.7757(8)$ & $-0.1069(10)$ & $0.048(3)$ \\
$\mathrm{C} 5$ & $0.556(3)$ & $0.8265(7)$ & $-0.0176(9)$ & $0.042(3)$ \\
$\mathrm{C} 6$ & $0.682(3)$ & $0.7978(7)$ & $0.0871(9)$ & $0.039(3)$ \\
$\mathrm{C} 7$ & $0.671(3)$ & $0.8383(7)$ & $0.1907(9)$ & $0.039(3)$ \\
$\mathrm{C} 8$ & $0.533(3)$ & $0.9191(7)$ & $0.2281(10)$ & $0.044(3)$ \\
$\mathrm{C} 9$ & $0.566(3)$ & $0.9430(8)$ & $0.3353(9)$ & $0.047(3)$ \\
$\mathrm{C} 10$ & $0.729(3)$ & $0.8854(8)$ & $0.4117(10)$ & $0.048(3)$ \\
$\mathrm{C} 11$ & $0.869(3)$ & $0.8032(8)$ & $0.3764(9)$ & $0.046(3)$ \\
$\mathrm{C} 12$ & $0.845(3)$ & $0.7785(8)$ & $0.2676(9)$ & $0.041(3)$ \\
$\mathrm{C} 13$ & $1.178(3)$ & $0.6333(8)$ & $0.2518(11)$ & $0.051(3)$ \\
$\mathrm{C} 14$ & $1.008(3)$ & $0.5530(9)$ & $0.2926(12)$ & $0.067(4)$ \\
$\mathrm{H} 3$ & 0.7995 & 0.6581 & -0.1623 & $0.0616^{*}$ \\
$\mathrm{H} 5$ & 0.4442 & 0.8811 & -0.0240 & $0.0498^{*}$ \\
$\mathrm{H} 8$ & 0.4181 & 0.9565 & 0.1791 & $0.0524^{*}$ \\
$\mathrm{H} 10$ & 0.7444 & 0.9015 & 0.4852 & $0.0581^{*}$ \\
$\mathrm{H} 13 \mathrm{~A}$ & 1.3166 & 0.6139 & 0.1951 & $0.0616^{*}$ \\
$\mathrm{H} 13 \mathrm{~B}$ & 1.3104 & 0.6590 & 0.3111 & $0.0616^{*}$ \\
$\mathrm{H} 14 \mathrm{~A}$ & 0.8767 & 0.5270 & 0.2342 & $0.0802^{*}$ \\
$\mathrm{H} 14 \mathrm{~B}$ & 0.8768 & 0.5712 & 0.3508 & $0.0802^{*}$ \\
$\mathrm{H} 14 \mathrm{C}$ & 1.1601 & 0.5084 & 0.3193 & \\
& & & & \\
\hline & & & & \\
\hline
\end{tabular}


Atomic displacement parameters $\left(\AA^{2}\right)$

\begin{tabular}{lllllll}
\hline & $U^{11}$ & $U^{22}$ & $U^{33}$ & $U^{12}$ & $U^{13}$ & $U^{23}$ \\
\hline Br1 & $0.0604(7)$ & $0.0431(7)$ & $0.0918(11)$ & $0.0099(7)$ & $0.0024(7)$ & $-0.0126(7)$ \\
Br2 & $0.0871(9)$ & $0.0752(10)$ & $0.0491(7)$ & $0.0044(9)$ & $-0.0140(7)$ & $-0.0013(7)$ \\
Br3 & $0.0759(8)$ & $0.0475(7)$ & $0.0738(9)$ & $0.0015(8)$ & $0.0042(7)$ & $-0.0187(7)$ \\
Br4 & $0.0710(9)$ & $0.0678(9)$ & $0.0557(8)$ & $-0.0063(8)$ & $-0.0143(7)$ & $0.0171(6)$ \\
N1 & $0.039(5)$ & $0.037(5)$ & $0.047(5)$ & $-0.009(4)$ & $-0.002(4)$ & $0.000(4)$ \\
C1 & $0.034(5)$ & $0.026(5)$ & $0.051(6)$ & $0.003(5)$ & $0.004(5)$ & $-0.004(5)$ \\
C2 & $0.031(5)$ & $0.040(6)$ & $0.066(8)$ & $-0.010(5)$ & $-0.001(5)$ & $0.003(5)$ \\
C3 & $0.052(6)$ & $0.048(7)$ & $0.055(7)$ & $-0.009(6)$ & $0.007(5)$ & $-0.017(6)$ \\
C4 & $0.050(6)$ & $0.040(6)$ & $0.054(7)$ & $-0.013(6)$ & $0.004(6)$ & $-0.006(5)$ \\
C5 & $0.049(6)$ & $0.020(5)$ & $0.054(7)$ & $-0.003(5)$ & $-0.011(5)$ & $0.002(4)$ \\
C6 & $0.037(5)$ & $0.033(6)$ & $0.046(6)$ & $-0.001(5)$ & $0.005(4)$ & $-0.002(5)$ \\
C7 & $0.038(5)$ & $0.030(5)$ & $0.048(6)$ & $-0.017(5)$ & $0.002(5)$ & $0.002(4)$ \\
C8 & $0.049(6)$ & $0.032(6)$ & $0.051(7)$ & $-0.007(5)$ & $0.001(5)$ & $0.002(5)$ \\
C9 & $0.057(7)$ & $0.048(6)$ & $0.037(6)$ & $-0.015(6)$ & $0.002(5)$ & $-0.004(5)$ \\
C10 & $0.054(6)$ & $0.042(6)$ & $0.049(7)$ & $-0.008(6)$ & $0.002(6)$ & $-0.007(5)$ \\
C11 & $0.047(6)$ & $0.047(7)$ & $0.043(6)$ & $-0.012(6)$ & $-0.009(5)$ & $0.007(5)$ \\
C12 & $0.036(5)$ & $0.041(6)$ & $0.047(6)$ & $-0.020(5)$ & $-0.002(5)$ & $0.004(5)$ \\
C13 & $0.039(6)$ & $0.037(6)$ & $0.076(9)$ & $0.002(5)$ & $-0.019(6)$ & $0.007(6)$ \\
C14 & $0.066(7)$ & $0.030(6)$ & $0.106(11)$ & $-0.004(7)$ & $0.013(7)$ & $0.018(7)$ \\
& & & & & & \\
\hline
\end{tabular}

Geometric parameters $\left(A,{ }^{\circ}\right)$

\begin{tabular}{llll}
\hline $\mathrm{Br} 1-\mathrm{C} 2$ & $1.912(11)$ & $\mathrm{C} 7-\mathrm{C} 12$ & $1.458(15)$ \\
$\mathrm{Br} 2-\mathrm{C} 4$ & $1.880(12)$ & $\mathrm{C} 8-\mathrm{C} 9$ & $1.359(16)$ \\
$\mathrm{Br} 3-\mathrm{C} 9$ & $1.894(12)$ & $\mathrm{C} 9-\mathrm{C} 10$ & $1.412(16)$ \\
$\mathrm{Br} 4-\mathrm{C} 11$ & $1.906(11)$ & $\mathrm{C} 10-\mathrm{C} 11$ & $1.418(16)$ \\
$\mathrm{N} 1-\mathrm{C} 1$ & $1.361(14)$ & $\mathrm{C} 11-\mathrm{C} 12$ & $1.379(15)$ \\
$\mathrm{N} 1-\mathrm{C} 12$ & $1.393(14)$ & $\mathrm{C} 13-\mathrm{C} 14$ & $1.476(17)$ \\
$\mathrm{N} 1-\mathrm{C} 13$ & $1.507(14)$ & $\mathrm{C} 3-\mathrm{H} 3$ & 0.930 \\
$\mathrm{C} 1-\mathrm{C} 2$ & $1.415(16)$ & $\mathrm{C} 5-\mathrm{H} 5$ & 0.930 \\
$\mathrm{C} 1-\mathrm{C} 6$ & $1.418(14)$ & $\mathrm{C} 8-\mathrm{H} 8$ & 0.930 \\
$\mathrm{C} 2-\mathrm{C} 3$ & $1.429(17)$ & $\mathrm{C} 10-\mathrm{H} 10$ & 0.930 \\
$\mathrm{C} 3-\mathrm{C} 4$ & $1.417(16)$ & $\mathrm{C} 13-\mathrm{H} 13 \mathrm{~A}$ & 0.970 \\
$\mathrm{C} 4-\mathrm{C} 5$ & $1.340(16)$ & $\mathrm{C} 13-\mathrm{H} 13 \mathrm{~B}$ & 0.970 \\
$\mathrm{C} 5-\mathrm{C} 6$ & $1.426(15)$ & $\mathrm{C} 14-\mathrm{H} 14 \mathrm{~A}$ & 0.960 \\
$\mathrm{C} 6-\mathrm{C} 7$ & $1.404(15)$ & $\mathrm{C} 14-\mathrm{H} 14 \mathrm{~B}$ & 0.960 \\
$\mathrm{C} 7-\mathrm{C} 8$ & $1.403(15)$ & $\mathrm{C} 14-\mathrm{H} 14 \mathrm{C}$ & \\
& & & $3.636(3)$ \\
$\mathrm{Br} 2 \cdots \mathrm{B} 44^{\mathrm{i}}$ & $3.660(3)$ & $\mathrm{Br} 3 \cdots \mathrm{B} 14 \mathrm{ii}$ & $125.5(9)$ \\
$\mathrm{C} 1-\mathrm{N} 1-\mathrm{C} 12$ & & $\mathrm{Br} 4-\mathrm{C} 11-\mathrm{C} 12$ & $120.3(10)$ \\
$\mathrm{C} 1-\mathrm{N} 1-\mathrm{C} 13$ & $110.4(8)$ & $\mathrm{C} 10-\mathrm{C} 11-\mathrm{C} 12$ & $106.2(9)$ \\
$\mathrm{C} 12-\mathrm{N} 1-\mathrm{C} 13$ & $125.6(9)$ & $\mathrm{N} 1-\mathrm{C} 12-\mathrm{C} 7$ & $135.2(10)$ \\
$\mathrm{N} 1-\mathrm{C} 1-\mathrm{C} 2$ & $123.8(9)$ & $\mathrm{N} 1-\mathrm{C} 12-\mathrm{C} 11$ & $118.5(10)$ \\
$\mathrm{N} 1-\mathrm{C} 1-\mathrm{C} 6$ & $134.0(9)$ & &
\end{tabular}




\begin{tabular}{|c|c|c|c|}
\hline $\mathrm{C} 2-\mathrm{C} 1-\mathrm{C} 6$ & $117.5(10)$ & $\mathrm{N} 1-\mathrm{C} 13-\mathrm{C} 14$ & $113.0(9)$ \\
\hline $\mathrm{Br} 1-\mathrm{C} 2-\mathrm{C} 1$ & $124.5(8)$ & $\mathrm{C} 2-\mathrm{C} 3-\mathrm{H} 3$ & 120.413 \\
\hline $\mathrm{Br} 1-\mathrm{C} 2-\mathrm{C} 3$ & $114.7(9)$ & $\mathrm{C} 4-\mathrm{C} 3-\mathrm{H} 3$ & 120.407 \\
\hline $\mathrm{C} 1-\mathrm{C} 2-\mathrm{C} 3$ & $120.7(10)$ & $\mathrm{C} 4-\mathrm{C} 5-\mathrm{H} 5$ & 119.576 \\
\hline $\mathrm{C} 2-\mathrm{C} 3-\mathrm{C} 4$ & $119.2(11)$ & $\mathrm{C} 6-\mathrm{C} 5-\mathrm{H} 5$ & 119.568 \\
\hline $\mathrm{Br} 2-\mathrm{C} 4-\mathrm{C} 3$ & $116.3(9)$ & $\mathrm{C} 7-\mathrm{C} 8-\mathrm{H} 8$ & 119.725 \\
\hline $\mathrm{Br} 2-\mathrm{C} 4-\mathrm{C} 5$ & $122.8(9)$ & $\mathrm{C} 9-\mathrm{C} 8-\mathrm{H} 8$ & 119.729 \\
\hline $\mathrm{C} 3-\mathrm{C} 4-\mathrm{C} 5$ & $120.9(11)$ & $\mathrm{C} 9-\mathrm{C} 10-\mathrm{H} 10$ & 119.964 \\
\hline $\mathrm{C} 4-\mathrm{C} 5-\mathrm{C} 6$ & $120.9(10)$ & $\mathrm{C} 11-\mathrm{C} 10-\mathrm{H} 10$ & 119.962 \\
\hline $\mathrm{C} 1-\mathrm{C} 6-\mathrm{C} 5$ & $120.9(10)$ & $\mathrm{N} 1-\mathrm{C} 13-\mathrm{H} 13 \mathrm{~A}$ & 108.986 \\
\hline $\mathrm{C} 1-\mathrm{C} 6-\mathrm{C} 7$ & $107.8(9)$ & $\mathrm{N} 1-\mathrm{C} 13-\mathrm{H} 13 \mathrm{~B}$ & 108.988 \\
\hline $\mathrm{C} 5-\mathrm{C} 6-\mathrm{C} 7$ & $131.3(9)$ & $\mathrm{C} 14-\mathrm{C} 13-\mathrm{H} 13 \mathrm{~A}$ & 108.984 \\
\hline $\mathrm{C} 6-\mathrm{C} 7-\mathrm{C} 8$ & $133.2(10)$ & $\mathrm{C} 14-\mathrm{C} 13-\mathrm{H} 13 \mathrm{~B}$ & 108.981 \\
\hline $\mathrm{C} 6-\mathrm{C} 7-\mathrm{C} 12$ & $106.9(9)$ & $\mathrm{H} 13 \mathrm{~A}-\mathrm{C} 13-\mathrm{H} 13 \mathrm{~B}$ & 107.779 \\
\hline $\mathrm{C} 8-\mathrm{C} 7-\mathrm{C} 12$ & $119.9(10)$ & $\mathrm{C} 13-\mathrm{C} 14-\mathrm{H} 14 \mathrm{~A}$ & 109.473 \\
\hline $\mathrm{C} 7-\mathrm{C} 8-\mathrm{C} 9$ & $120.5(10)$ & $\mathrm{C} 13-\mathrm{C} 14-\mathrm{H} 14 \mathrm{~B}$ & 109.466 \\
\hline $\mathrm{Br} 3-\mathrm{C} 9-\mathrm{C} 8$ & $122.1(9)$ & $\mathrm{C} 13-\mathrm{C} 14-\mathrm{H} 14 \mathrm{C}$ & 109.472 \\
\hline $\mathrm{Br} 3-\mathrm{C} 9-\mathrm{C} 10$ & $117.2(9)$ & $\mathrm{H} 14 \mathrm{~A}-\mathrm{C} 14-\mathrm{H} 14 \mathrm{~B}$ & 109.470 \\
\hline $\mathrm{C} 8-\mathrm{C} 9-\mathrm{C} 10$ & $120.6(11)$ & $\mathrm{H} 14 \mathrm{~A}-\mathrm{C} 14-\mathrm{H} 14 \mathrm{C}$ & 109.470 \\
\hline $\mathrm{C} 9-\mathrm{C} 10-\mathrm{C} 11$ & $120.1(11)$ & $\mathrm{H} 14 \mathrm{~B}-\mathrm{C} 14-\mathrm{H} 14 \mathrm{C}$ & 109.476 \\
\hline $\mathrm{Br} 4-\mathrm{C} 11-\mathrm{C} 10$ & $114.2(8)$ & & \\
\hline $\mathrm{C} 1-\mathrm{N} 1-\mathrm{C} 12-\mathrm{C} 7$ & $-2.6(10)$ & $\mathrm{C} 3-\mathrm{C} 4-\mathrm{C} 5-\mathrm{C} 6$ & $-0.3(16)$ \\
\hline $\mathrm{C} 1-\mathrm{N} 1-\mathrm{C} 12-\mathrm{C} 11$ & $178.7(10)$ & $\mathrm{C} 4-\mathrm{C} 5-\mathrm{C} 6-\mathrm{C} 1$ & $-0.2(15)$ \\
\hline $\mathrm{C} 12-\mathrm{N} 1-\mathrm{C} 1-\mathrm{C} 2$ & $-179.2(9)$ & $\mathrm{C} 4-\mathrm{C} 5-\mathrm{C} 6-\mathrm{C} 7$ & $-179.9(9)$ \\
\hline $\mathrm{C} 12-\mathrm{N} 1-\mathrm{C} 1-\mathrm{C} 6$ & $3.2(10)$ & $\mathrm{C} 1-\mathrm{C} 6-\mathrm{C} 7-\mathrm{C} 8$ & $-179.3(9)$ \\
\hline $\mathrm{C} 1-\mathrm{N} 1-\mathrm{C} 13-\mathrm{C} 14$ & $-92.7(12)$ & $\mathrm{C} 1-\mathrm{C} 6-\mathrm{C} 7-\mathrm{C} 12$ & $0.9(10)$ \\
\hline $\mathrm{C} 13-\mathrm{N} 1-\mathrm{C} 1-\mathrm{C} 2$ & $4.9(16)$ & $\mathrm{C} 5-\mathrm{C} 6-\mathrm{C} 7-\mathrm{C} 8$ & $0.4(18)$ \\
\hline $\mathrm{C} 13-\mathrm{N} 1-\mathrm{C} 1-\mathrm{C} 6$ & $-172.6(8)$ & $\mathrm{C} 5-\mathrm{C} 6-\mathrm{C} 7-\mathrm{C} 12$ & $-179.4(9)$ \\
\hline $\mathrm{C} 12-\mathrm{N} 1-\mathrm{C} 13-\mathrm{C} 14$ & $91.9(11)$ & $\mathrm{C} 6-\mathrm{C} 7-\mathrm{C} 8-\mathrm{C} 9$ & $-178.4(10)$ \\
\hline $\mathrm{C} 13-\mathrm{N} 1-\mathrm{C} 12-\mathrm{C} 7$ & $173.4(8)$ & $\mathrm{C} 6-\mathrm{C} 7-\mathrm{C} 12-\mathrm{N} 1$ & $1.0(10)$ \\
\hline $\mathrm{C} 13-\mathrm{N} 1-\mathrm{C} 12-\mathrm{C} 11$ & $-5.3(17)$ & $\mathrm{C} 6-\mathrm{C} 7-\mathrm{C} 12-\mathrm{C} 11$ & $179.9(8)$ \\
\hline $\mathrm{N} 1-\mathrm{C} 1-\mathrm{C} 2-\mathrm{Br} 1$ & $6.9(16)$ & $\mathrm{C} 8-\mathrm{C} 7-\mathrm{C} 12-\mathrm{N} 1$ & $-178.9(8)$ \\
\hline $\mathrm{N} 1-\mathrm{C} 1-\mathrm{C} 2-\mathrm{C} 3$ & $-176.1(9)$ & $\mathrm{C} 8-\mathrm{C} 7-\mathrm{C} 12-\mathrm{C} 11$ & $0.1(14)$ \\
\hline $\mathrm{N} 1-\mathrm{C} 1-\mathrm{C} 6-\mathrm{C} 5$ & $177.7(8)$ & $\mathrm{C} 12-\mathrm{C} 7-\mathrm{C} 8-\mathrm{C} 9$ & $1.4(15)$ \\
\hline $\mathrm{N} 1-\mathrm{C} 1-\mathrm{C} 6-\mathrm{C} 7$ & $-2.5(10)$ & $\mathrm{C} 7-\mathrm{C} 8-\mathrm{C} 9-\mathrm{Br} 3$ & $179.4(8)$ \\
\hline $\mathrm{C} 2-\mathrm{C} 1-\mathrm{C} 6-\mathrm{C} 5$ & $-0.3(13)$ & $\mathrm{C} 7-\mathrm{C} 8-\mathrm{C} 9-\mathrm{C} 10$ & $-2.4(16)$ \\
\hline $\mathrm{C} 2-\mathrm{C} 1-\mathrm{C} 6-\mathrm{C} 7$ & $179.4(8)$ & $\mathrm{Br} 3-\mathrm{C} 9-\mathrm{C} 10-\mathrm{C} 11$ & $-179.7(7)$ \\
\hline $\mathrm{C} 6-\mathrm{C} 1-\mathrm{C} 2-\mathrm{Br} 1$ & $-175.7(7)$ & $\mathrm{C} 8-\mathrm{C} 9-\mathrm{C} 10-\mathrm{C} 11$ & $2.0(16)$ \\
\hline $\mathrm{C} 6-\mathrm{C} 1-\mathrm{C} 2-\mathrm{C} 3$ & $1.3(13)$ & $\mathrm{C} 9-\mathrm{C} 10-\mathrm{C} 11-\mathrm{Br} 4$ & $-178.9(9)$ \\
\hline $\mathrm{Br} 1-\mathrm{C} 2-\mathrm{C} 3-\mathrm{C} 4$ & $175.5(7)$ & $\mathrm{C} 9-\mathrm{C} 10-\mathrm{C} 11-\mathrm{C} 12$ & $-0.5(16)$ \\
\hline $\mathrm{C} 1-\mathrm{C} 2-\mathrm{C} 3-\mathrm{C} 4$ & $-1.7(15)$ & $\mathrm{Br} 4-\mathrm{C} 11-\mathrm{C} 12-\mathrm{N} 1$ & $-3.7(17)$ \\
\hline $\mathrm{C} 2-\mathrm{C} 3-\mathrm{C} 4-\mathrm{Br} 2$ & $-178.4(8)$ & $\mathrm{Br} 4-\mathrm{C} 11-\mathrm{C} 12-\mathrm{C} 7$ & $177.7(6)$ \\
\hline $\mathrm{C} 2-\mathrm{C} 3-\mathrm{C} 4-\mathrm{C} 5$ & $1.2(16)$ & $\mathrm{C} 10-\mathrm{C} 11-\mathrm{C} 12-\mathrm{N} 1$ & $178.1(10)$ \\
\hline $\mathrm{Br} 2-\mathrm{C} 4-\mathrm{C} 5-\mathrm{C} 6$ & $179.4(6)$ & $\mathrm{C} 10-\mathrm{C} 11-\mathrm{C} 12-\mathrm{C} 7$ & $-0.5(15)$ \\
\hline
\end{tabular}

Symmetry codes: (i) $x-1, y, z-1$; (ii) $-x+1, y+1 / 2,-z+1$. 